\title{
Analysis of the vibration characteristics of double-walled carbon nanotubes
}

\author{
Toshiaki Natsuki, Qing-Qing Ni \\ Faculty of Textile Sicence \& Technology, Shinshu University, \\ 3-15-1 Tokida, Ueda-shi, 386-8567, Japan
}

Morinobu Endo

Faculty of Engineering, Shinshu University,

4-17-1 Wakasato, Nagano-shi, 380-8553, Japan

\begin{abstract}
The induced electric-field has been applied to measure the elastic modulus of carbon nanotubes. However, the vibrational modes of the multi-walled carbon nanotubes are quite different from those of the single-walled carbon nanotubes. Analysis of the vibration characteristics of double-walled carbon nanotubes (DWCNTs) with simply supported boundary condition are carried out based on Euler-Bernoulli beam theory. The DWCNTs are considered as two nanotube shells coupled through the van der Waals interaction between them. It is found that the vibrational modes of DWCNTs are noncoaxial intertube vibrations, and the deflections of the inner and outer nanotubes can occur in the same or in opposite deflections. In the same vibrational mode, the resonant frequencies of DWCNTs with deflections between the inner and outer nanotubes in the same direction are smaller than those of DWCNTs with the opposite deflections.
\end{abstract}

\footnotetext{
*Corresponding author: TEL: 081-0268-21-5421; $\quad$ FAX: 81-0268-21-5482

E-mail: natsuki@shinshu-u.ac.jp (T.Natsuki)
} 


\section{Introduction}

The discovery of carbon nanotubes (CNTs) has stimulated extensive research activities in science and engineering devoted to carbon nanostructures and nanocomposites. Many studies have shown that the carbon nanotubes possess remarkable mechanical and physical properties [1-4] leading to many potential applications such as fluid transport, fluid storage at nanoscale, and nanodevices for drug delivery $[5,6]$. Since controlled experiments at the nanometer scale are very difficult, the simulation techniques have been widely and successfully used to investigate the mechanical property, wave propagation and resonant frequency of nanoscale materials [7-12]. It is well known that a molecular dynamics (MD) method is an effective method to simulate the properties of materials with microstructures. However, the MD simulation is very time-consuming and remains formidable for large-scale systems. Therefore, simulation and computation of larger systems is currently left to continuum mechanics methods [13]. The study of vibrational properties in CNTs is of great current interest, which can be used to measure the elastic modules of individual CNTs $[14,15]$. In some early works on the vibrational properties in CNTs, the role of van der Waals (vdW) force interaction between two adjacent nanotubes in non-coaxial resonance for MWCNTs has been studied using the multiple-Euler-beam model $[12,16]$. They assumed that all nested individual nanotubes had the same vibrational mode. The results indicated that noncoaxial resonance would be excited at the higher natural frequencies. Moreover, other studies of the vibrational property and the wave propagation in MWCNTs have been focused on Flügge shell model [9,10]. Li et. al. [17,18] have presented vibrational analysis of single- and double-walled CNTs using a truss rod model. They predicted that the single-walled nanotubes (SWCNTs) could have fundamental frequency ranging from $10 \mathrm{GHz}$ to $1.5 \mathrm{THz}$ and the vibrational frequency depends on the diameter and length of nanotubes [17].

In this paper, the main objective is to propose a theoretical approach to vibration 
characteristic analysis of CNTs with simply-supported boundary condition. For double-walled nanotubes (DWCNTs), the interaction between the inner and outer nanotubes is considered to be coupled together through the vdW force. Using the present analytical method, we can easy to obtain the resonant frequencies of DWCNTs with different vibrational modes. The analytical method could be used to determine the elastic modulus of CNTs through measuring a few fundamental resonant frequencies.

\section{Theoretical approach}

The continuum mechanics method has been successfully applied to analyze the dynamic responses of individual carbon nanotubes. Based on the Euler-Bernoulli beam model, the equation of motion of a free vibration in CNTs is given by

$$
\rho A \frac{\partial^{2} w}{\partial^{2} t}+E I \frac{\partial^{4} w}{\partial^{4} x}=p
$$

where $x$ and $t$ are the axial coordinate and time, respectively. $w(x, t)$ is the deflection of

CNTs. $p$ is the distributed transverse force acted on the CNTs. $E$ and $I$ are the elastic modulus and the moment of inertia of a cross-section, respectively. $A$ is the cross-sectional area and $\rho$ is the mass density of CNTs.

For the DWCNTs, the deflection of nested tubes is considered to be coupled together through vdW force between the inner and outer nanotubes. The interaction pressure at any point between any two adjacent tubes depends linearly on the difference of their deflections at that point. Based on the Euler-Bernoulli beam model, therefore, the differential equations governing the fluid motion are described by the following two-coupled equations:

$$
\begin{aligned}
& \rho A_{1} \frac{\partial^{2} w_{1}}{\partial^{2} t}+E I_{1} \frac{\partial^{4} w_{1}}{\partial^{4} x}=c\left(w_{2}-w_{1}\right) \\
& \rho A_{2} \frac{\partial^{2} w_{2}}{\partial^{2} t}+E I_{2} \frac{\partial^{4} w_{2}}{\partial^{4} x}=-c\left(w_{2}-w_{1}\right)
\end{aligned}
$$

where the subscripts 1 and 2 denote the quantities associated with the inner and outer 
nanotubes, respectively. $c$ is the vdW interaction coefficient between nanotubes. In this simulation, the vdW interaction energy potential can be estimated as [19]

$$
c=\frac{\pi \varepsilon R_{1} R_{2} \sigma^{6}}{a^{4}}\left[\frac{1001 \sigma^{6}}{3} H^{13}-\frac{1120}{9} H^{7}\right],
$$

where

$$
H^{m}=\left(R_{1}+R_{2}\right)^{-m} \int_{0}^{\pi / 2} \frac{d \theta}{\left(1-K \cos ^{2} \theta\right)^{m / 2}}, \quad(m=7,13)
$$

and

$$
K=\frac{4 R_{1} R_{2}}{\left(R_{1}+R_{2}\right)^{2}} .
$$

where $\sigma$ and $\varepsilon$ are the vdW radius and the well depth of the Lennard-Jones potential, respectively. $a=0.142 \mathrm{~nm}$ is the carbon-carbon bond length. $R_{1}$ and $R_{2}$ are the inner and outer radius, respectively.

In this analysis, we consider the deflection of DWCNTs has different vibrational modes $Y_{j}(x), j=1,2$ for the inner and outer nanotubes. The displacements of the vibrational solution in DWCNTs can be given by

$$
w_{j}=Y_{j}(x) e^{i \omega t}, \quad(j=1,2),
$$

where $\omega$ is the vibrational frequency.

Substituting Eq. (7) into Eqs. (2) and (3), we obtain the coupled equation of the vibrational properties in DWCNTs:

$$
\left[\begin{array}{cc}
c-\rho A_{1} \omega^{2}+E I_{1} D^{(4)} & -c \\
-c & c-\rho A_{2} \omega^{2}+E I_{2} D^{(4)}
\end{array}\right]\left\{\begin{array}{l}
Y_{1} \\
Y_{2}
\end{array}\right\}=\left\{\begin{array}{l}
0 \\
0
\end{array}\right\}
$$

where $D$ is the differential operator. Eliminating one of the two variables $Y_{1}$ and $Y_{2}$ from Eq. (8), we yield

$$
\left(D^{(8)}+2 p D^{(4)}+q\right) W=0
$$

where $W=Y_{1}, Y_{2}$, 
and

$$
\begin{aligned}
& p=\frac{c}{2}\left(\frac{1}{E I_{1}}+\frac{1}{E I_{2}}\right)-\frac{\rho \omega^{2}}{2}\left(\frac{A_{1}}{E I_{1}}+\frac{A_{2}}{E I_{2}}\right) \\
& q=\frac{\rho^{2} A_{1} A_{2}-\rho c\left(A_{1}+A_{2}\right) \omega^{2}}{\left(E I_{1}\right)\left(E I_{2}\right)}
\end{aligned}
$$

If $p^{2}>q$ and $q<0$, the solutions of the differential equation (9) are expressed as

$$
\begin{aligned}
Y_{1} & =C_{1} e^{\sqrt[4]{\alpha} x}+C_{2} e^{-\sqrt[4]{\alpha} x}+C_{3} \cos (\sqrt[4]{\alpha} x)+C_{4} \sin (\sqrt[4]{\alpha} x) \\
& +e^{(\sqrt[4]{\beta} / \sqrt{2}) x}\left[C_{5} \cos (\sqrt[4]{\beta} / \sqrt{2}) x+C_{6} \sin (\sqrt[4]{\beta} / \sqrt{2}) x\right]+e^{-(\sqrt[4]{\beta} / \sqrt{2}) x}\left[C_{7} \cos (\sqrt[4]{\beta} / \sqrt{2}) x+C_{8} \sin (\sqrt[4]{\beta} / \sqrt{2}) x\right]
\end{aligned}
$$

$$
\begin{aligned}
Y_{2} & =\lambda_{1} C_{1} e^{\sqrt[4]{\alpha} x}+\lambda_{1} C_{2} e^{-\sqrt[4]{\alpha} x}+\lambda_{1} C_{3} \cos (\sqrt[4]{\alpha} x)+\lambda_{1} C_{4} \sin (\sqrt[4]{\alpha} x) \\
& +\lambda_{2} e^{(\sqrt[4]{\beta} / \sqrt{2}) x}\left[C_{5} \cos (\sqrt[4]{\beta} / \sqrt{2}) x+C_{6} \sin (\sqrt[4]{\beta} / \sqrt{2}) x\right]+\lambda_{2} e^{-(\sqrt[4]{\beta} / \sqrt{2}) x}\left[C_{7} \cos (\sqrt[4]{\beta} / \sqrt{2}) x+C_{8} \sin (\sqrt[4]{\beta} / \sqrt{2}) x\right]
\end{aligned}
$$

where $C_{j}(j=1,2, \cdots, 8)$ are unknown integration constants and

$$
\alpha=-p+\sqrt{p^{2}-q}, \quad \beta=p+\sqrt{p^{2}-q}
$$

and $\lambda_{1}$ and $\lambda_{2}$ are associated amplitude ratios of the outer to inner nanotubes, which can be obtained from Eq. (8)

$$
\lambda_{j}=1+\frac{E I_{1} \beta_{j}^{4}}{c}-\frac{\rho A_{1} \omega^{2}}{c}, \quad j=1,2
$$

where $\beta_{1,2}=-p \pm \sqrt{p^{2}-q}$.

If $p^{2}>q>0$ and $p<0$, the solution of Eq. (9) is given by

$$
\begin{aligned}
Y_{1}= & C_{1} e^{\sqrt[4]{\alpha} x}+C_{2} e^{-\sqrt[4]{\alpha} x}+C_{3} \cos (\sqrt[4]{\alpha} x)+C_{4} \sin (\sqrt[4]{\alpha} x) \\
& +C_{5} e^{\sqrt[4]{\beta} x}+C_{6} e^{-\sqrt[4]{\beta} x}+C_{7} \cos (\sqrt[4]{\beta} x)+C_{8} \sin (\sqrt[4]{\beta} x) \\
Y_{2}= & \lambda_{1} C_{1} e^{\sqrt[4]{\alpha} x}+\lambda_{1} C_{2} e^{-\sqrt[4]{\alpha} x}+\lambda_{1} C_{3} \cos (\sqrt[4]{\alpha} x)+\lambda_{1} C_{4} \sin (\sqrt[4]{\alpha} x) \\
& +\lambda_{2} C_{5} e^{\sqrt[4]{\beta} x}+\lambda_{2} C_{6} e^{-\sqrt[4]{\beta} x}+\lambda_{2} C_{7} \cos (\sqrt[4]{\beta} x)+\lambda_{2} C_{8} \sin (\sqrt[4]{\beta} x)
\end{aligned}
$$

Substituting Eqs. (12)-(17) into a given boundary condition, the simultaneous equation can be 
obtained as follows:

$$
M\{\alpha, \beta, L\}_{8 \times 8}\left[\begin{array}{c}
C_{1} \\
C_{2} \\
\ldots \\
C_{8}
\end{array}\right]=0,
$$

where $M\{\alpha, \beta, L\}_{8 \times 8}$ is a $8 \times 8$ matrix with elements of $\alpha, \beta, L$. The condition for nonzero solutions of $C_{j}(j=1,2, \cdots, 8)$ is $|M|_{8 \times 8}=0$, which can be used to analyze the vibrational frequencies of DWCNTs.

For free frequency of DWCNTs with simply supported-simply supported and with a length of $L$, the boundary conditions are

$$
\begin{aligned}
& Y_{1}(0)=Y_{1}(L)=Y_{1}^{\prime \prime}(0)=Y_{1}^{\prime \prime}(L)=0 \\
& Y_{2}(0)=Y_{2}(L)=Y_{2}^{\prime \prime}(0)=Y_{2}^{\prime \prime}(L)=0
\end{aligned}
$$

For the vibration of DWCNTs with clamped-clamped supported boundary conditions, we have

$$
\begin{aligned}
& Y_{1}(0)=Y_{1}(L)=Y_{1}^{\prime}(0)=Y_{1}^{\prime}(L)=0 \\
& Y_{2}(0)=Y_{2}(L)=Y_{2}^{\prime}(0)=Y_{2}^{\prime}(L)=0
\end{aligned}
$$

The boundary conditions of cantilevered DWCNTs are

$$
\begin{aligned}
& Y_{1}(0)=Y_{1}^{\prime}(0)=Y_{1}^{\prime \prime}(L)=Y_{1}^{\prime \prime \prime}(L)=0 \\
& Y_{2}(0)=Y_{2}^{\prime}(0)=Y_{2}^{\prime \prime}(L)=Y_{2}^{\prime \prime \prime}(L)=0
\end{aligned}
$$

\section{Numerical results and discussion}

In this study, vibration characteristic analysis was carried out for DWCNT with simply supported-simply supported boundary condition. According to the transmission electron microscopy (TEM) observation of DWCNT reported by Iijima [20], the inner and outer diameters of a DWCNT are $4.8 \mathrm{~nm}$ and $5.5 \mathrm{~nm}$, respectively. And also, the thickness of each 
layer in the DWCNT was observed to be that of a graphite sheet with $0.34 \mathrm{~nm}$. The CNTs had an elastic modulus of 1.0 TPa, Poisson's ratio of 0.27 , and the density of $2.0 \mathrm{~g} / \mathrm{cm}^{3}$. The vdW parameters in the Lennard-Jones potential are taken as $\varepsilon=2.967 \mathrm{meV}$ and $\sigma=0.34 \mathrm{~nm}$ as reported recently by Saito et al. [21]. As an example, first, a numerical result was compared with a solution of Yoon et al. [12]. They found that the natural frequency is close that given by the single-beam model. For assuming a DWCNT with aspect ratio of 20, the inner and the outer diameters of 0.7 and $1.4 \mathrm{~nm}$, respectively, the natural frequency is $0.35 \mathrm{THz}$ and the intertube resonant frequency is about $10 \mathrm{THz}$ which is insensitive to the mode number. According to the present model, the natural frequency of DWCNTs, about $0.62 \mathrm{THz}$ for the fist mode, is largely affected by the mode numbers. The vibrational frequency of DWCNTs is between the vibrational solutions of the inner and outer nanotubes obtained from existing model.

Figure 1 shows six noncoaxial vibrational modes of the DWCNTs with simply supportedsimply supported boundary condition based on the present theoretical approach. It is found that all modes exhibit noncoaxial intertube vibrations of DWCNTs. Moreover, the vibrational amplitudes of the inner nanotube are larger than those of the outer nanotube. For the fundamental or first mode, the vibrational mode is almost coaxial because the amplitude ratio between the inner and outer nanotubes is very close to one. The DWCNT with the 2 th mode is two half-waves and the deflections of the inner and outer nanotubes are in the same direction. In the case of the 3th mode, the vibrational mode is one half-wave but the deflection of the outer nanotube is opposite to the deflection of the inner nanotube. The noncoaxial intertube vibration would crucially affect some of their important physical properties. The DWCNT with the 4th mode exhibits two half-waves, and the vibrational deflection between the inner and outer nanotubes is in the opposite deflection. In the case of the 5th and 6th modes, the characteristics of both vibrational modes are three half-waves, but the vibrational amplitudes 
of DWCNTs with the 5th and 6th modes have the same and opposite deflections, respectively.

Figure 2 shows the frequency responses associated with different vibrational modes (see Fig. 1) for both SCNTs and DWCNTs. In the case of SCNTs, the natural frequency of the SCNTs with different vibrational modes can be given by

$$
\omega_{i}=\frac{\beta_{i}}{L^{2}} \sqrt{\frac{E I}{\rho S}}
$$

where $\beta_{1}=\pi^{2}, \beta_{2}=4 \pi^{2}$ and $\beta_{3}=9 \pi^{2}$ are mode values corresponding to the one, two and three half-waves, respectively. $S$ is the cross-sectional area of CNTs.

It is found that from Fig. 2 that the SCNTs have a larger vibrational frequency than the DWCNTs increased by at least $30 \%$. In the case of DWCNTs, the characteristic frequencies are largely affected by the vibrational deflection of the inner and outer nanotubes. The vibrational frequencies of DWCNTs with the opposite deflection are larger than those of the opposite deflection. It has been well known that the vibrational frequency of tubes increases with increasing harmonic numbers. According to our simulations, however, it is worthy of notice that the vibrational frequency of DWCNTs with the first harmonic can be higher than that of the second harmonic. This is because the vibrational deflection of the inner to outer nanotubes is in the opposite direction.

The vibrational frequencies of DWCNTs with different lengths are shown in Figure 3 as the function of the vibrational modes. It is found that the DWCNTs exhibit the vibrational frequency over terahertz. The vibrational frequency with the first harmonic (the fundamental mode) is about $2.1 \mathrm{THz}$ for the DWCNTs with the length of $10 \mathrm{~nm}$. The resonant frequencies of DWCNTs decrease with increasing length of nanotubes.

\section{Conclusions}

A theoretical approach based on a multiple-elastic-beam model is proposed to study the 
vibrational properties in DWCNTs with simply supported-simply supported boundary condition. This method gives good calculate the vibrational frequency of DWCNTs because the inner and outer nanotubers are considered to have different vibrational modes. Using the present theoretical approach, the noncoaxial resonant frequencies of DWCNTs with different vibrational modes are investigated. The vibrational amplitudes of the inner and nanotubes are noncoaxial and can have opposite deflection. In the case of an identical harmonic mode, the vibrational frequency of DWCNTs is larger for the opposite deflection than for the same deflection. The resonant frequencies decrease with increasing length of nanotubes.

\section{Acknoledgements}

This work was supported by CLUSTER (the second stage) of Ministry of Education, Culture, Sports Science and Technology (Japan). 


\section{References}

[1] Thostenson ET, Ren Z, Chou TW. Advances in the science and technology of carbon nanotubes and their composites: a review. Comp Sci Tech 2001;61:1899-1912.

[2] Schadler LS, Giannaris SC, Ajayan PM. Load transfer in carbon nanotube epoxy composites. Appl Phys Lett 1998;73(26):3842-3844.

[3] Vaccarini L, Goze C, Henrard L, Hernandez E, Bernier P, Rubio A. Mechanical and electronic properties of carbon and born-nitride nanotubes. Carbon 2000;38:1681-1690.

[4] Yu MF, Lourie O, Dyer MJ, Moloni K, Kelly TF, Ruoff RS. Strength and breaking mechanism of multi-walled carbon nanotubes under tensile load. Science 2000;287(28): 637-640.

[5] Nadarajan SB, Katsikis PD, Papazoglou ES. Loading carbon nanotubes with viscous fluids and nanoparticles - a simpler approach, Applied Physics A 2007;89(2):437-442.

[6] Dalton AB, Collins S, Razal J, Munoz E, Ebron V.H, Kim BG., et al., Baughman RH. Continuous carbon nanotube composite fibers: properties, potential applications, and problems. J Mater Chem 2004;14(1):1-3.

[7] Natsuki T, Tantrakarn K, Endo M. Prediction of elastic properties for single-walled carbon nanotubes Carbon 2004;42(1):39-45.

[8] Li C, Chou TW. Elastic wave velocities in single-walled carbon nanotubes. Phys Rev B 2006;73(24):245407.

[9] Natsuki T, Ni QQ, Endo M. Wave propagation in single- and double-walled carbon nanotubes filled with fluids. J Appl Phys 2007;101(3):034319.

[10] Natsuki T, Endo M, Tsuda H. Vibration analysis of embedded carbon nanotubes using wave propagation approach. J Appl Phys 2006;99(3):034311.

[11] Sun C, Liu K.Vibration of multi-walled carbon nanotubes with initial axial loading. Solid State Commun 2007;143 (4-5):202-207. 
[12] Yoon J, Ru CQ, Mioduchowski A. Noncoaxial resonance of an isolated multiwall carbon nanotube. Phys Rev B 2002;66(23):2334021-2334024

[13] Qian D, Wagner G.J, Liu WK, Yu MF, Ruoff RS. Mechanics of carbon nanotubes. Appl Mech Rev 2002;55(6):495-532.

[14] Treacy MMJ, Ebbesen TW, Gibson JM. Exceptionally high Young's modulus observed for individual carbon nanotubes. Nature 1996;381:678-680.

[15] Krishnan A, Dujardin E, Ebbesen TW, Yianilos PN, Treacy MMJ. Young's modulus of single-walled nanotubes. Phys Rev B 1998;58(20):14013-14019.

[16] Yoon J, Ru CQ, Mioduchowski A. Vibration of an embedded multiwall carbon nanotube composites. Science and Technology 2003;63 (11):1533-1542.

[17] Li C, Chou TW. Single-walled carbon nanotubes as ultrahigh frequency nanomechanical resonators. Phys Rev B 2003;68(7):734051-734053.

[18] Li C, Chou TW, Vibrational behaviors of multiwalled-carbon-nanotube-based nanomechanical resonators. Appl Phys Lett 2004;84(1):121-123.

[19] He XQ, Kitipornchai S, Wang CM, Liew KM. Modeling of van der Waals force for infinitesimal deformation of multi-walled carbon nanotubes treated as cylindrical shells. Int J Solids Struct 2005;42(23):6032-6047.

[20] Lijima S. Helical microtubules of graphitic carbon. Nature 1991;354:56-58.

[21] Saito R., Matsuo R, Kimura T, Dresselhaus G., Dresselhaus MS. Anomalous potential barrier of double-wall carbon nanotube. Chem Phys Lett 2001;348(3-4):187-193. 


\section{Figure captions}

Figure 1 Six noncoaxial vibrational modes of DWCNTs with simply supported-simply supported boundary condition

Figure 2 Vibrational frequencies in SCNT and DWCNT for six vibrational modes (nanotube length of $20 \mathrm{~nm}$ )

Figure 3 Relationship between vibrational frequencies and vibrational modes for DWCNTs with simply supported 
Fig.1 Six noncoaxial vibrational modes of DWCNTs with simply supported-simply supported boundary condition

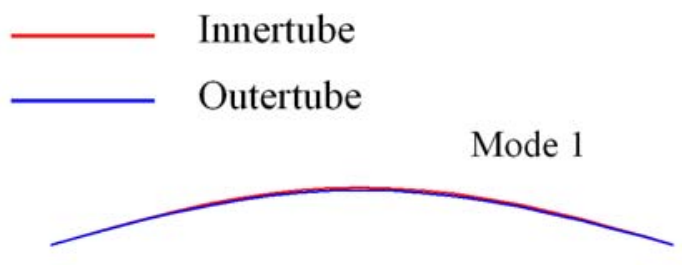

Mode 4

Mode 2

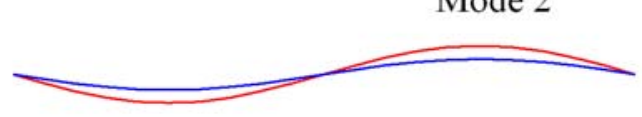

Mode 3
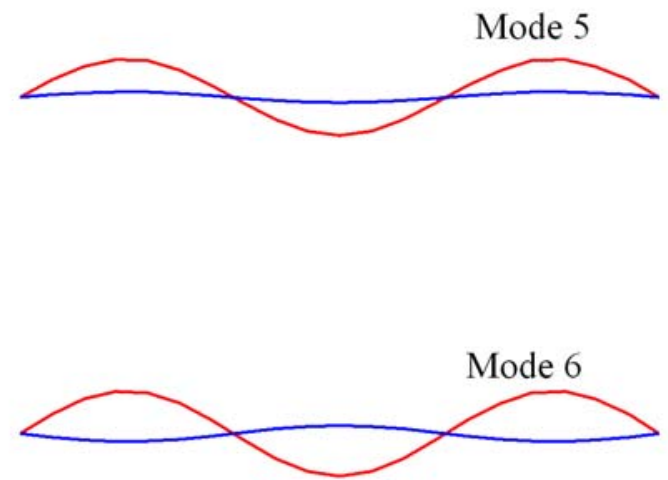
Figure 2 Vibrational frequencies in SCNT and DWNT for six vibrational modes (nanotube length of $20 \mathrm{~nm})$

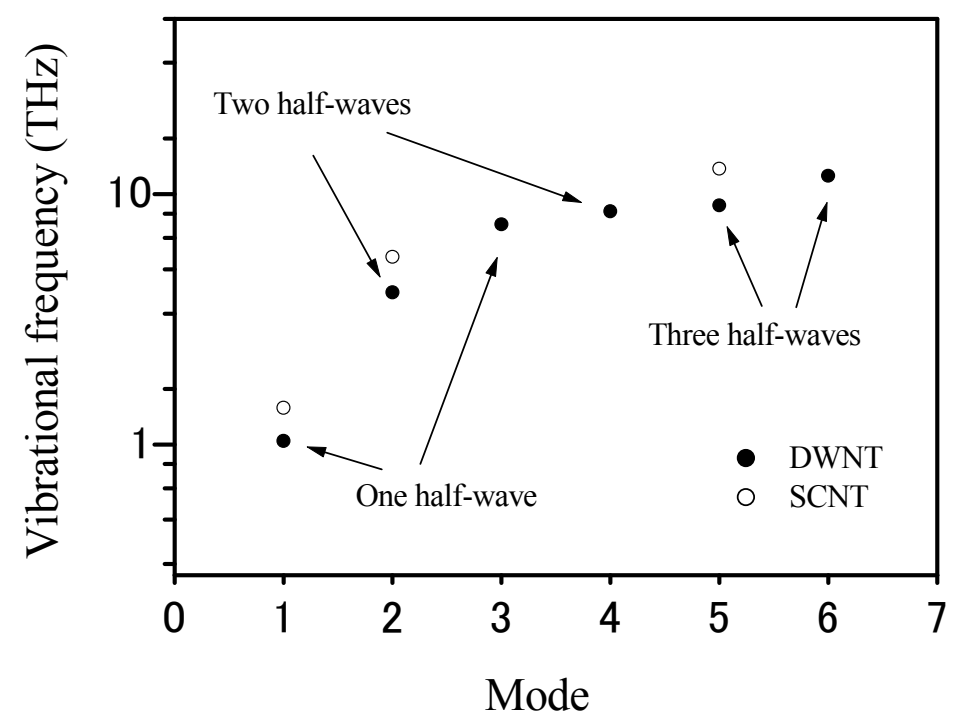


Figure 3 Relationship between vibrational frequencies and vibrational modes for DWCNTs with simply supported

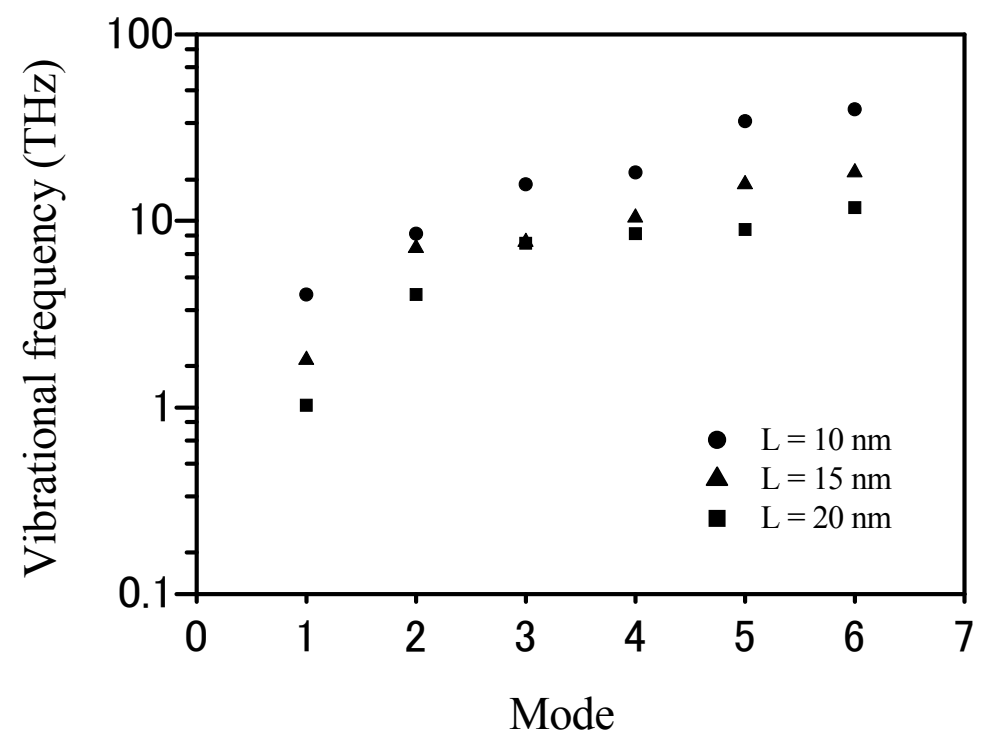

